\title{
Christina Horvath, Le roman urbain contemporain en France
}

\section{Gabriella Bosco}

\section{Q OpenEdition \\ 1 Journals}

\section{Edizione digitale}

URL: http://journals.openedition.org/studifrancesi/8430

DOI: ERREUR PDO dans /localdata/www-bin/Core/Core/Db/Db.class.php L.34 : SQLSTATE[HY000]

[2006] MySQL server has gone away

ISSN: 2421-5856

\section{Editore}

Rosenberg \& Sellier

\section{Edizione cartacea}

Data di pubblicazione: 1 mai 2009

Paginazione: 227

ISSN: 0039-2944

\section{Notizia bibliografica digitale}

Gabriella Bosco, «Christina Horvath, Le roman urbain contemporain en France», Studi Francesi [Online], 157 (LIII | I) | 2009, online dal 30 novembre 2015, consultato il 07 janvier 2021. URL: http://

journals.openedition.org/studifrancesi/8430 ; DOI: https://doi.org/10.4000/studifrancesi.8430

Questo documento è stato generato automaticamente il 7 janvier 2021.

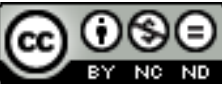

Studi Francesi è distribuita con Licenza Creative Commons Attribuzione - Non commerciale - Non opere derivate 4.0 Internazionale. 


\title{
Christina Horvath, Le roman urbain contemporain en France
}

\author{
Gabriella Bosco
}

\section{NOTIZIA}

CHRISTINA HORVATH, Le roman urbain contemporain en France, Paris, Presses Sorbonne

Nouvelle, 2007, pp. 260.

1 Basato sull'analisi di una trentina di romanzi pubblicati tra il 1989 e il 2001, il saggio di Christina HORVATH delinea le caratteristiche di quello che - alla luce della nozione di surmodernité: "une modernité extrême et contemporaine, liée aux changements d'échelle, à l'émergence des non-lieux et à l'abolissement de la distance entre les grandes métropoles grâce aux moyens de transports rapides» - può essere considerato un nuovo genere, il romanzo urbano. In che modo, si chiede l'A., quello che è generalmente considerato il genere dominante nella letteratura del xx secolo riesce a rendere conto di fenomeni quali la mondializzazione, la globalizzazione o l'iperconcentrazione urbana? Con quali tecniche riesce a trasformarli in fiction? Doppio lo scopo perseguito: esaminare la produzione romanzesca francese contemporanea per descrivere le modificazioni in essa riscontrabili rispetto al genere e ai procedimenti narrativi; e porre le basi di una teoria generale del romanzo urbano in quanto espressione caratteristica di un'epoca.

2 Il decennio preso in considerazione rappresenta un arco di tempo sufficientemente ampio per l'analisi. I romanzi scelti hanno in comune il fatto di situare la loro trama in una grande metropoli occidentale e di distinguersi dal resto della produzione letteraria per il loro carattere urbano: il corpus comprende romanzi di Olivier Adam, Frédéric Beigbeder, Tonino Benacquista, Emmanuèle Bernheim, Jean-Yves Cendrey, Marie Darrieussecq, Philippe Delerm, Agnès Desarthe, Virginie Despentes, Jean Echenoz, Christian Gailly, Michel Houelle-becq, Tassadit Imache, Jean-Claude Izzo, Nicolas JoneGorlin, Eric Laurrent, Eric Le Braz, Mathieu Lindon, Marie Ndiaye, Christian Oster, 
Daniel Pennac, Lydie Salvayre, Paul Smaïl, Dominique Souton, Jean-Philippe Toussaint e Cécile Wajsbrot. Di alcuni autori è preso in considerazione più di un titolo.

3 I dodici capitoli del saggio sono organizzati lungo tre assi principali: i primi quattro analizzano il rapporto che il romanzo intrattiene con la città interrogandosi sul modo in cui lo spazio urbano vi si fa testo; i capitoli dal quinto al nono si concentrano sui personaggi caratteristici del romanzo urbano e sul tipo di trama che essi generano; gli ultimi quattro capitoli trattano invece dell'interferenza e dell'interazione all'interno delle opere studiate di diversi tipi di discorso (letterario, giornalistico, televisivo, pubblicitario, cinematografico, ecc.) che coesistono nella cultura eterogenea delle megalopoli.

Il quadro che risulta riflette l'immagine di un genere volto alla rappresentazione dell'universo sociale delle città reali. La città non vi è percepita come mostruosità incomprensibile né come figura fantasmatica. Al contrario, il romanzo urbano mira a fornire una descrizione molto precisa della vita quotidiana ordinaria nel contesto cittadino, interessandosi sia ai destini individuali sia all'attività politica, sociale e culturale dell'epoca contemporanea. A differenza di altre narrazioni che a loro volta ricorrono ad ambientazioni urbane ma senza farne la struttura portante, il romanzo urbano elegge la città a personaggio principale. E così facendo si rivela come la forma più adeguata a tradurre in testo il presente. 